\title{
Evolution of Twin Structure in Cold-rolled Copper
}

\author{
Q. H. Lu, M. L. Sui and D. X. Li
}

Shenyang National Laboratory for Materials Science, Institute of Metal Research, Chinese Academy of Sciences, 72 Wenhua Road, Shenyang 110016, China

Growth twins were often observed in the Cu samples, especially for the electrodeposited $\mathrm{Cu}$ [1], because of the lower stacking-fault energy in copper. The existence of twins might affect the slipping behavior during deformation. However, there were less research works on the effect of the twins during deformation. In this work, a kind of electrodeposited copper rich of twins was cold rolled and studied by means of high-resolution transmission electron microscopy (HRTEM). The evolution of twin structure during deformation was presented.

A bulk copper plate with purity of $99.993 \%$ was prepared by means of electrodeposition method [2]. Specimens with a thickness of $1 \mathrm{~mm}$ were cut off from the copper plate along the direction perpendicular to the plate, and then cold rolled to the elongation of about $160 \%$ at room temperature. Figure 1(a) is a typical TEM image of the as-deposited samples observed along the direction perpendicular to the deposited plate. Most of grains with a size of submicrometer were contained many twins in a layer structure with a thickness ranged from several to a few decade nanometers. Figure 1(b) is a HRTEM image of the twin layers observed along [110]. The twin boundaries indicated by arrowhead are smooth with only a few dislocations. Figure 2(a) is the morphology of the twins in the as-rolled samples observed along [110]. It was clear that the twin boundaries were widened and the numbers of twin step and dislocation were increased. From the HRTEM image of these twins, as shown in Fig. 2(b), it can be seen been that there is a kind of periodic contrast at the twin boundary, which was caused by the overlap of the twin-related crystals [3]. Obviously, the twin boundaries were moved during cold rolling. The movement of twin boundaries was performed by the slipping of a $1 / 6<112>$ Shockley dislocation on the $\{111\}$ plane. It should be mentioned that the thickness of the twins in the electrodeposited $\mathrm{Cu}$ is in the range of nanometer, which is comparable with the dislocation-free distance in $\mathrm{Cu}(\sim 19.3 \mathrm{~nm})[4]$. The existence of these twins would suppress the slipping on the $<111>$ planes across the twin boundaries and enhance the slipping on the $<111>$ planes parallel with the twin boundaries. That is, the slipping of $1 / 6<112>$ Shockley dislocations on the twin boundaries would be very active, which induced some layers were thickened and their twins were narrowed. The twins were took an important role in plastic deformation of the electrodeposited copper [5].

\section{References}

[1] A.Gangulee, J. Appl. Phys 3, (1972) 867.

[2] L. Lu et al, Science 287, (2000) 1463.

[3] E. Abe et al, Phil. Mag A 75, (1997) 975.

[4] T. G. Nieh and J. Wadsworth, J. Wadsworth, Scripta. Matll. Mater 25(1991) 955.

[5] This research was supported by NSFC on the grant No 50125103, 50271074 and 90206044. 

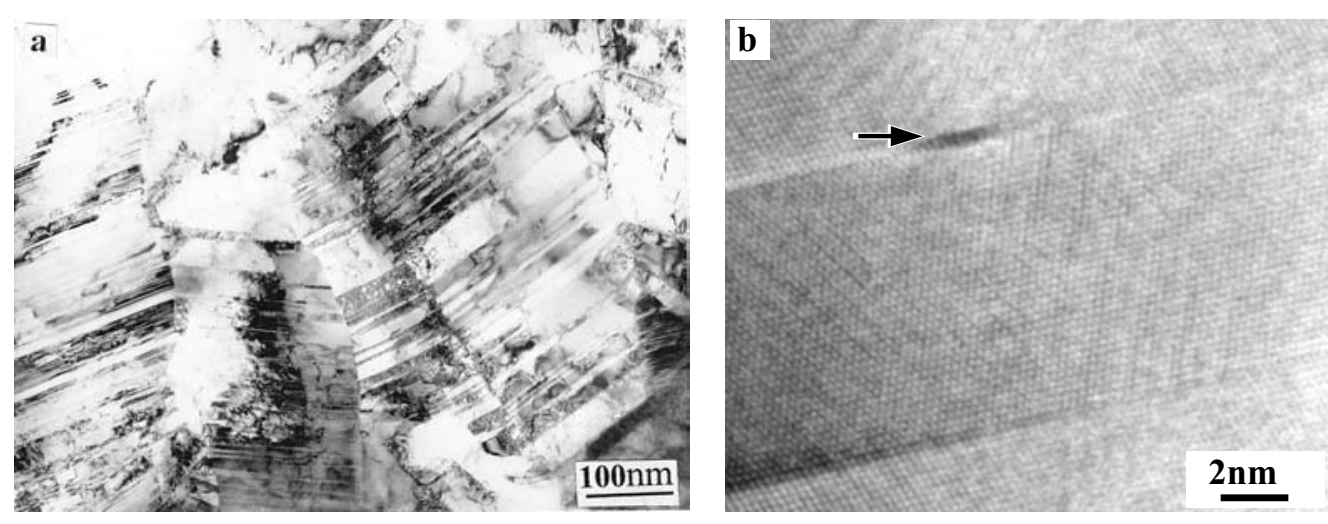

Figure 1. TEM image of twins (a) and HRTEM image of the twin boundary in as-deposited $\mathrm{Cu}$, respectively.
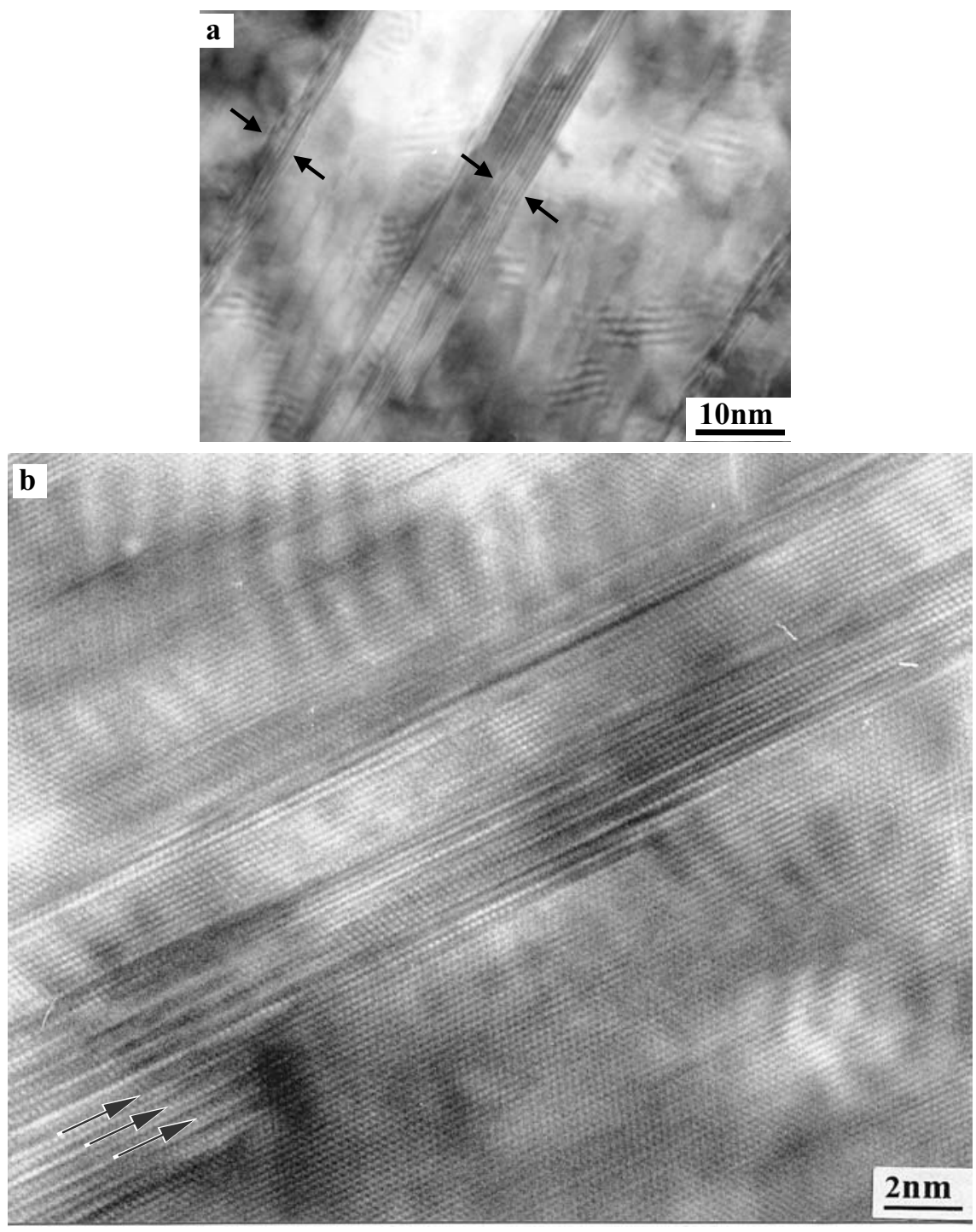

Figure 2. TEM image of twin layers (a) and HRTEM image of twins in as-rolled $\mathrm{Cu}$ cold-rolled to $160 \%$, respectively. 